\title{
A Meta-Analytic Review of Verbal Fluency Performance Following Focal Cortical Lesions
}

\author{
Julie D. Henry and John R. Crawford \\ King's College, University of Aberdeen
}

\begin{abstract}
A meta-analysis of 31 studies with 1,791 participants was conducted to investigate the sensitivity of tests of verbal fluency to the presence of focal cortical lesions. Relative to healthy controls, participants with focal frontal injuries had large and comparable deficits on phonemic $(r=.52)$ and semantic $(r=.54)$ fluency. For frontal but not nonfrontal patients, phonemic fluency deficits qualified as differential deficits when compared with IQ and psychomotor speed; phonemic fluency was also more strongly and more specifically related to the presence of frontal lesions than the Wisconsin Card Sorting Test scores. In contrast, temporal damage was associated with a lesser deficit on phonemic fluency $(r=.44)$ but a larger deficit on semantic fluency $(r=.61)$.
\end{abstract}

Tests of verbal fluency are among the most widely used measures to assess cognitive functioning after neurological damage. These measures require time-restricted generation of multipleresponse alternatives under constrained search conditions and involve associative exploration and retrieval of words based on phonemic or semantic criteria (phonemic and semantic fluency, respectively). Verbal fluency tests, in particular phonemic fluency, are commonly used to assess executive dysfunction (Crawford \& Henry, in press; Parker \& Crawford, 1992; Phillips, 1997; R. M. Reitan \& Wolfson, 1994).

Executive functioning is thought to be responsible not for basic cognitive processes but for the set of behavioral competencies that integrate these capacities (Stuss \& Benson, 1986). This aspect of cognition, therefore, permits contextually sensitive, flexible responses. Because all cognitive tests involve a strategic component to at least a certain extent, defining precisely what constitutes an executive measure has proven problematic. However, cognitive tasks particularly dependent on executive processes are presumed to impose substantial demands on self-directed planning and strategy formation (Stuss \& Benson, 1986) and require future-oriented, goal-directed (Welsh, Satterlee-Cartmell, \& Stine, 1999), and nonhabitual responses (Perret, 1974; Phillips, 1997).

Phonemic fluency is regarded as a measure of executive dysfunction because generating words on the basis of orthographic criteria is unusual, requiring the creation of nonhabitual strategies based primarily on lexical representations (Perret, 1974). In addition, the measure requires efficient organization of verbal retrieval and recall as well as self-monitoring aspects of cognition (the participant must keep track of responses already given), effortful self-initiation, and inhibition of responses when appropriate (Crawford \& Henry, in press; Perret, 1974; Phillips, 1997; Ruff, Light, Parker, \& Levin, 1997). Although these cognitive demands are consistent with most definitions of executive functioning, the extent to which semantic fluency should be regarded as a measure

Julie D. Henry and John R. Crawford, Department of Psychology, King's College, University of Aberdeen, Aberdeen, Scotland.

Correspondence concerning this article should be addressed to Julie D. Henry, Department of Psychology, King's College, University of Aberdeen, Aberdeen AB24 3HN, Scotland. E-mail: j.d.henry@abdn.ac.uk of this construct remains less clear. Searching for semantic extensions of a target superordinate relies on well-established search strategies consistent with the organizational structure of the world. Thus, deficits may reflect problems with semantic memory, not executive dysfunction.

Neuropathologically, there is a great deal of evidence that the neural substrates of executive processes lie in the frontal cortex (see Stuss \& Benson, 1986). Thus, because frontal lesions are considered to be particularly associated with executive deficits, for a measure to be regarded as "executive," an important means of cross-validation is to demonstrate that patients with frontal damage are impaired on it (Phillips, 1997). However, empirical studies have found verbal fluency deficits to be associated with clinical conditions that vary in terms of the relative prominence of frontal dysfunction, including schizophrenia (Crawford, Obonsawin, \& Bremner, 1993), traumatic brain injury (Brooks, Fos, Greve, \& Hammond, 1999), Parkinson's disease (Flowers, Robertson, \& Sheridan, 1995), and depression (Crowe, 1996). Moreover, patients with focal nonfrontal injuries have also been found to perform poorly on tests of verbal fluency (Loring, Meador, \& Lee, 1994; R. C. Martin, Loring, Meador, \& Lee, 1990).

It is not surprising that tests of verbal fluency are sensitive to brain damage per se because patients with severe neurological problems are often impaired on virtually all measures of cognition. However, this lack of specificity means that demonstrating that a particular measure is sensitive to frontal damage is not sufficient by itself to infer that a deficit on the measure in question reflects executive dysfunction. Instead, it must be shown that frontal patients are more impaired on tests of verbal fluency than nonfrontal patients and, more importantly, that frontal but not nonfrontal patients exhibit a differential fluency deficit (i.e., a deficit in excess of the average performance deficit across a range of other cognitive tasks that are not considered to impose heavy executive demands).

Evidence of a differential deficit is particularly important given that, as with all cognitive measures, tests of verbal fluency are multifactorial. Miller's (1984) study demonstrated how fluency deficits in different clinical conditions may not reflect a common underlying problem. Patients with focal frontal lesions, dementia of the Alzheimer's type (DAT), and controls were administered a 
test of phonemic fluency. Although all clinical groups were significantly impaired relative to controls, when predicted fluency scores were derived from measures of verbal IQ (VIQ), there was a substantial discrepancy between predicted and actual scores for the frontal groups but not for those with DAT. Thus, although performance levels in DAT were consistent with a generalized cognitive deficit, frontal patients exhibited a differential deficit that could not be attributed to decrements in VIQ, thereby suggesting a specific problem with executive processing.

Thus, when assessing the implications of verbal fluency deficits, it is critical that patients' general level of intellectual functioning be taken into consideration (Crawford \& Henry, in press), especially because brain-injured patients with greater than average intelligence have been found to outperform below-average controls with no evidence of cerebral trauma on this measure (Borkowski, Benton, \& Spreen, 1967). However, it is particularly important to ascertain whether deficits on measures of verbal fluency exceed those for VIQ. Verbal fluency tests have a substantial verbal component, and, indeed, phonemic fluency was originally developed as a measure of VIQ (Thurstone \& Thurstone, 1941). Correlations of .64 (Crawford et al., 1993), .67 (Crawford, Moore, \& Cameron, 1992), and even 87 (Miller, 1984) have been reported between phonemic fluency and VIQ. Thus, deficits on tests of verbal fluency do not by themselves provide evidence of executive dysfunction; rather, it is the pattern of deficits across fluency versus VIQ (and to a lesser extent full-scale IQ [FSIQ]) that is important. Many have emphasized the importance of assessing whether deficits on executive measures qualify as differential deficits (Crawford, Bryan, Luszcz, Obonsawin, \& Stewart, 2000; Laws, 1999; Miller, 1984), and a rigorous assessment of this issue is critical if the construct validity of tests of verbal fluency as executive measures is to be upheld.

As noted earlier, it is also unclear whether phonemic and semantic fluency can be regarded as comparable in terms of their relative dependence on executive processes. It is, therefore, important to also address whether these two types of fluency are equivalent in terms of their relative dependence on different cortical structures. Phonemic fluency is considered to be particularly sensitive to left frontal damage (Benton, 1968; Milner, 1964; Perret, 1974; Stuss et al., 1998; Tucha, Smely, \& Lange, 1999), which Ramier and Hacean (1970) have attributed to the operation of two basic factors: a verbal factor that is usually located in the left hemisphere and an executive factor that reflects the integrity of the frontal lobes. However, it has been argued that, relative to phonemic fluency, the demands imposed by semantic fluency on executive processes are substantially reduced (Perret, 1974).

Perret (1974) argued that the two tasks differ qualitatively because only phonemic fluency requires suppressing the habitual behavior of using words in a manner related to their meaning. Because semantic fluency search constraints are consistent with the normal organizational structure of the lexicon, these particular inhibitory mechanisms are not required. However, empirical support for Perret's (1974) perspective is inconsistent. Although Baldo and Shimamura (1998) reported that, irrespective of laterality, both phonemic and semantic fluency were significantly, and comparably, impaired in frontal patients relative to healthy controls, other studies suggest that phonemic fluency may be more sensitive to frontal damage than semantic fluency. Beldarrain, Grafman, Pascual-Leone, and Garcia-Monco (1999), for example, found that patients with frontal lesions were significantly impaired on phonemic but not semantic fluency.

It has also been suggested that, relative to phonemic fluency, semantic fluency imposes greater demands on semantic memory, the component of long-term memory that contains the permanent representation of our knowledge of objects, facts, and concepts as well as words and their meaning (Hodges, Salmon, \& Butters, 1992). Semantic memory is, therefore, critical for the identification and naming of objects and the understanding and production of written words. Because there is a great deal of evidence that temporal structures are the neural substrates particularly responsible for semantic memory, semantic fluency should be relatively more dependent on these cortical regions than phonemic fluency.

Consistent with this perspective, in a functional imaging study, Gourovitch et al. (2000) compared regional cerebral blood flow patterns during performance of phonemic and semantic fluency tests in a sample of healthy volunteers. They found that, relative to a control task, the two measures were associated with comparable activation patterns, including increased activation in left prefrontal regions. However, when phonemic and semantic fluency were directly compared, the left temporal cortex was revealed to be activated more during semantic than phonemic fluency. In addition, temporal structures are disproportionately affected in the early stages of DAT (Terry \& Katzman, 1983), and there is a strong trend for patients with this disorder to be more impaired on semantic than phonemic fluency (Monsch et al., 1997).

Phonemic and semantic fluency are also differentially disrupted by specific secondary interference tasks. Martin, Wiggs, Lalonde, and Mack (1994), for example, found that, although a secondary task hypothesized to activate the temporal lobes (object decision) significantly disrupted semantic but not phonemic fluency performance in a sample of healthy volunteers, a frontal lobe-activating secondary task (sequence tapping) was associated with the opposite pattern of impairment. This double dissociation suggests that there is a relatively greater contribution of the frontal lobes to phonemic fluency performance, but that temporal functioning is particularly critical to semantic fluency. Finally, Troyer, Moscovitch, Winocur, Alexander, and Stuss (1998) found that, relative to controls, patients with unilateral left temporal lesions did not differ on phonemic fluency but were significantly impaired on semantic fluency. However, other studies have reported a significant relationship between temporal damage and both types of fluency (Loring et al., 1994; R. C. Martin et al., 1990).

Stuss et al. (1998) argue that inconsistencies between studies regarding the relative dependence of phonemic and semantic fluency on different cortical structures may reflect differences in chronicity, precise lesion localization, cause, age, and aphasia, as well as the nature of the comparison group and the precise dependent measure used. Although we have considerable sympathy with this view, it is also necessary to entertain the possibility that some of the variability is artifactual. Hunter, Schmidt, and Jackson (1982) identified many sources of artificial variation across studies, and, when reviewing certain topics in educational research, took an extreme position: "It is our experience that there is usually no important variation in study results after sampling error and other artefacts are removed" (p. 32). Because there is a tendency for sample size to be relatively small in clinical studies, this area of research is especially susceptible to reification of sampling error, the most serious source of artifactual variance. However, it is not 
possible to correct for this artifact at the level of the individual study (Hunter \& Schmidt, 1994).

Our study is, to our knowledge, the first to use meta-analytic techniques to assess the effect of focal cortical lesions on verbal fluency performance. One of the most important advantages of this methodology is that corrections can be implemented for sampling error. Thus, in the current study, it will be possible to assess whether discrepancies between studies reflect the influence of substantive factors or artifactual variance.

The first aim is to derive mean effect size estimates for phonemic and semantic fluency, stratified according to lesion location (frontal, nonfrontal, and, specifically, temporal) and laterality (left vs. right). The magnitude of each of these means will provide a quantitative summary of the extent to which focal lesions in different cortical regions correlate with performance on each of the fluency measures. As noted, the principal neural substrates of executive processes and semantic memory are presumed to lie in the frontal and temporal lobes, respectively. By quantifying the magnitude of deficits on each of these measures for patients with lesions restricted to each of these cortical structures, the relative dependence of each on executive processes and semantic memory, respectively, can be assessed.

Evidence suggests that phonemic fluency and semantic fluency may not impose comparable demands on executive processes and semantic memory. Thus, the second aim is to compare quantitatively the mean effect sizes for phonemic versus semantic fluency for patients with focal temporal and focal frontal lesions.

Another issue relates to whether the verbal fluency deficits for each of these lesion groups reflect executive or generalized dysfunction (i.e., whether fluency deficits qualify as differential deficits). Thus, the third aim is to compare effects for both general IQ (FSIQ) and VIQ as measured by the Wechsler Adult Intelligence Scales (Wechsler, 1955, 1981) with the corresponding verbal fluency deficits for patients with frontal and nonfrontal lesions.

Relatedly, effect sizes are calculated for the Trail Making Test-Part A (TMT-A; W. Reitan, 1990), a widely used measure of psychomotor speed. A comparison of the magnitude of the deficits associated with this measure with that for verbal fluency is used to address the possibility that deficits on tests of verbal fluency simply reflect generalized slowing (i.e., a reduction in cognitive speed) rather than executive dysfunction.

Finally, effect sizes are calculated for the number of categories completed and perseverative errors on the Wisconsin Card Sorting Test (WCST-CC and WCST-PE, respectively; Heaton, 1981), one of the most widely used measures of executive functioning (Crawford \& Henry, in press; Phillips, 1997; R. M. Reitan \& Wolfson, 1994). A comparison of verbal fluency with the WCST will permit an examination of the convergent validity of these putative measures of executive function as well as an assessment of their relative sensitivity and specificity to frontal and nonfrontal dysfunction.

\section{Method}

\section{Sample of Studies}

A manual search of issues of Neuropsychology, Clinical Neuropsychologist, Journal of the International Neuropsychological Society, Neuropsychiatry, Neuropsychology and Behavioural Neurology, Journal of Neuropsychiatry and Clinical Neurosciences, Neuropsychologia, and Journal of Clinical and Experimental Neuropsychology was conducted. A computer-based search involving the Web of Science, Psych Lit CD-ROM, and Science Direct databases was also undertaken, using the following terms as search parameters: letter fluency, FAS, semantic fluency, category fluency, controlled oral word association, COWA(T), word fluency, verbal fluency, oral fluency, phonemic fluency, executive test, and frontal test. The search was completed in October 2002

Several inclusion criteria had to be met. First, the patient group had to consist entirely of adults with focal lesions localized in the frontal, temporal, or nonfrontal cortex or lateralized to either the right or left hemisphere. Membership of lesion location and laterality groups was not mutually exclusive. In addition, the study had to include a healthy control group free from neurological or psychiatric disease or signs of neurological or psychiatric disease (i.e., studies that included "pseudoneurological" control groups were also excluded; Beldarrain et al., 1999; Bolter, Long, \& Wagner, 1983). Each study also had to include a measure of phonemic or semantic fluency. Effect size estimates for FSIQ, VIQ, WCST-CC, WCST$\mathrm{PE}$, and TMT-A were derived from studies that also included verbal fluency. If both FSIQ and VIQ were reported in the same study, VIQ was preferentially coded.

For inclusion, the study must also have presented precise statistics convertible to effect size $r$. Thus, some studies were excluded because it was not possible to calculate effect sizes from the information provided (e.g., Levine et al., 1998; Stuss et al., 1998; Stuss, Gallup, \& Alexander, 2001) or only imprecise effect sizes could be calculated (Mangels, Ivry, \& Shimizu, 1998; Perret, 1974). Finally, studies had to have been published in English in a journal.

\section{Statistical Analysis}

Meta-analysis is a rigorous alternative to the traditional review process because it involves statistical integration of results. The basis of this methodology is the effect size, a standardized statistic that quantifies the magnitude of an effect. In the current study, the effect size $r$ was used, which corresponds to the degree of correlation between group membership (i.e., presence or absence of a focal cortical lesion) and performance on the measure of interest. The correlation coefficient is associated with a slight bias; thus, Fisher (1928) derived a transformation of $r$ that Snedecor and Cochran (1989) recommended be used in preference to $r$. However, this transformed estimate is itself associated with a bias, and in a Monte Carlo analysis, Field (2001) reported that transformed effect size estimates produced substantial upward biases of a larger magnitude than the corresponding downward biases associated with untransformed correlation coefficients. Thus, in the current study, untransformed correlation coefficients have been used for statistical analyses.

For each construct, effects were pooled to derive an estimate of the mean; each effect was weighted for sample size to correct for sampling error. To do so, the random-effects meta-analytic model (Shadish \& Haddock, 1994) was selected in preference to the more commonly used fixed-effects model because it yields more generalizable parameter estimates. This is because, in the fixed-effects model, the mean is presumed to reflect a common underlying effect parameter. However, in the random-effects model, the mean represents a hyperparameter because it allows for substantive differences beyond sampling error that differentiate the effects contributing to each respective mean (Raudenbush, 1994).

Statistically, the crucial difference between these methodologies is in the calculation of standard errors and confidence intervals, which for the random-effects model are typically larger. The National Research Council (1992) argues that the fixed-effects model should be the exception rather than the rule because it may lead to inappropriately strong conclusions. Thus, although more technically demanding than the fixed-effects model, it 
was considered important to use the random-effects model in the current work.

It was also important to test whether the difference in the magnitude of mean effects between, for instance, frontal and nonfrontal patients on phonemic fluency was statistically significant. However, there is no agreed-on method for statistically comparing mean effects using the random-effects meta-analytic model. A particular difficulty is whether the degrees of freedom in such analyses should be based on the number of participants or the number of studies. Given that a relatively small number of studies contribute to each respective mean in the current work, it was considered important to maximize the sensitivity to detect differences in mean effects. Therefore, the standardized difference between the two mean effects of interest was calculated using total sample size as the degrees of freedom.

Occasionally, the same participants contributed toward the mean effect for both variables to be compared (e.g., in comparisons of frontal patients' performance on phonemic vs. semantic fluency). Although each participant only contributed once when determining the sample size used for inferential statistics, including particular groups more than once violates the assumption of statistical independence of effect sizes. In addition, although meta-analysis reduces the impact of sampling error, this methodology also introduces another potential source of error when, as is the case in the current study, there may be duplication of patients or other participants in studies coming from the same experimental location or the same group of scientists. There is, unfortunately, no elegant way to deal with these problems.

Mean effects were also calculated for each of the nonfluency variables identified (IQ, WCST-CC, WCST-PE, and TMT-A) and compared with phonemic fluency. Because there were relatively few effects for each of these nonfluency measures, when comparing each nonfluency mean effect with phonemic fluency, for each comparison the mean effect for phonemic fluency was recalculated using only those studies that assessed both the nonfluency measure of interest as well as phonemic fluency. Semantic fluency was not included in these analyses because few studies were eligible.

To interpret how important a particular effect was in practical terms, we adopted J. Cohen's (1977) guidelines. These suggest that a correlation of .1 should be regarded as representing a small effect, .3 as medium, and .5 as large. In addition, effect sizes were squared and multiplied by 100 because these represent the percentage of the variance $(P V)$ on a measure of interest that is accounted for by group membership (i.e., having a focal cortical lesion vs. being a member of the healthy adult population).

Finally, multiple effect sizes for the same construct were permitted from the same study when more than one experiment was included in the study, or subgroups were created within a particular experiment so long as each respective group differed from one another in terms of the participants sampled. Thus, a particular summary statistic (including effect size estimates) may be based on a number of different groups of participants that exceeds the total number of studies.

\section{Results}

\section{Participant Characteristics}

Thirty-one studies published between 1982 and 2002 met the inclusion criteria specified. In total, data from 995 focal patients and 796 controls were included. Although relatively closely matched on demographic variables, there was a slight tendency for patients to be younger than controls $(M=49.85, S D=10.35$ vs. $M=51.40, S D=12.21$, respectively), less educated $(M=12.12$, $S D=2.08$ vs. $M=12.67, S D=2.43$, respectively), and male (57.74\% vs. $53.99 \%$, respectively).

\section{Effect Sizes Stratified According to Lesion Location and Laterality}

Study-level effect sizes for phonemic and semantic fluency broken down according to lesion location and laterality are presented in Appendix A. For effect sizes, a positive value indicates that patients have performed worse than controls, and a negative value indicates the opposite.

Table 1 presents estimates of the mean effects, their variability, and indexes of their practical importance. To estimate the degree of heterogeneity of the effects contributing to each mean, the homogeneity statistic $(Q)$ and the random effects variance $\left(\sigma_{\theta}^{2}\right)$ were also estimated, as well as the standard deviation of random effects and the $95 \%$ confidence intervals (CIs) within which random effects can be expected to fall. $Q$ quantifies within-group heterogeneity (i.e., the degree to which the studies contributing to each respective mean can be regarded as homogenous). If the $Q$ statistic associated with a mean effect is significant, this suggests that there are substantive differences between the studies contributing to that particular mean. In contrast, a nonsignificant estimate of $Q$ suggests that, once sampling error has been removed, no substantive differences between the studies contributing to the respective mean in question remain (i.e., the null hypothesis of homogeneity of effects cannot be rejected).

All mean effects were significantly different from zero and, in terms of practical importance, all were at least small to medium in magnitude. The $P V$ accounted for by group membership ranged from $6.8 \%$ to $44.9 \%$. For phonemic fluency, the mean deficit for the total frontal group $(r=.52)$ was significantly larger than for the total nonfrontal group $(r=.33, Z=4.13, p<.01)$ but did not differ significantly from patients with specifically temporal lesions ( $r=.44, Z=1.50, p=.13)$. The phonemic fluency deficits associated with left frontal $(r=.60)$, left nonfrontal $(r=.44)$, and left focal $(r=.56)$ damage were significantly larger than the corresponding deficits for unilateral right frontal $(r=.39$, $Z=3.77, p<.01)$, right nonfrontal $(r=.26, Z=2.12, p=.03)$, and right focal damage $(r=.37, Z=4.28, p<.01)$, respectively.

Nonfrontal patients $(r=.55)$ were comparably impaired on semantic fluency relative to frontal patients $(r=.54)$. Although temporal patients $(r=.61)$ were more impaired on semantic fluency than frontal patients, this difference did not attain significance $(Z=0.99, p=.32$ ). However, both nonfrontal and temporal patients were significantly more impaired on semantic fluency than on phonemic fluency ( $r \mathrm{~s}=.55$ vs. $.33, Z=2.85, p=$ $.01 ; r s=.61$ vs. $.44, Z=1.99, p=.05$, respectively). As for phonemic fluency, although both hemispheres were implicated in performance, lesions lateralized to the left hemisphere were particularly disruptive ( $r \mathrm{~s}=.67 \mathrm{vs} . .47$ for unilateral left and unilateral right, respectively; $Z=3.45, p<.01$ ). This basic pattern was also apparent for patients with left frontal $(r=.66)$ compared with right frontal $(r=.48)$ lesions and left nonfrontal $(r=.64)$ compared with right nonfrontal $(r=.44)$ lesions, although, because of low statistical power, neither of these comparisons attained significance $(Z=1.92, p=.06$, and $Z=1.75, p=.08$, respectively).

The main results can be more readily appreciated by referring to Figure 1. It can be seen that, although the deficits for phonemic and semantic fluency are comparable for the total frontal group as 
Table 1

Performance as a Function of Lesion Location and Laterality Relative to Healthy Controls

\begin{tabular}{|c|c|c|c|c|c|c|c|c|c|c|c|c|c|}
\hline \multirow[b]{2}{*}{ Lesion location and laterality } & \multirow[b]{2}{*}{$M$} & \multirow[b]{2}{*}{$K$} & \multirow[b]{2}{*}{$N$} & \multirow[b]{2}{*}{$S E$} & \multicolumn{2}{|c|}{$\begin{array}{c}95 \% \mathrm{CI} \\
(M)\end{array}$} & \multirow[b]{2}{*}{$Z$} & \multirow[b]{2}{*}{$P V$} & \multirow[b]{2}{*}{$O$} & \multirow[b]{2}{*}{$\sigma_{\theta}^{2}$} & \multirow[b]{2}{*}{$S D$} & \multicolumn{2}{|c|}{$\begin{array}{c}95 \% \text { CI } \\
\text { (mean effects) }\end{array}$} \\
\hline & & & & & Lower & Upper & & & & & & Lower & Upper \\
\hline \multicolumn{14}{|c|}{ Phonemic fluency } \\
\hline Frontal, total & .52 & 36 & 495 & .028 & .46 & .57 & $18.6^{*}$ & 27.0 & $73.2^{*}$ & .013 & .115 & .29 & .74 \\
\hline Unilateral left & .60 & 12 & 156 & .040 & .52 & .68 & $14.9 *$ & 35.9 & $21.5^{*}$ & .009 & .094 & .41 & .78 \\
\hline Unilateral right & .39 & 12 & 171 & .047 & .30 & .48 & $8.3^{*}$ & 15.0 & 16.9 & .009 & .093 & .20 & .57 \\
\hline Nonfrontal, total & .33 & 14 & 209 & .071 & .19 & .47 & $4.7 *$ & 11.1 & $70.7 *$ & .055 & .234 & -.13 & .79 \\
\hline Unilateral left & .44 & 6 & 92 & .104 & .24 & .64 & $4.2 *$ & 19.4 & $27.4 *$ & .050 & .224 & .00 & .88 \\
\hline Unilateral right & .26 & 6 & 91 & .123 & .02 & .50 & $2.1 *$ & 6.8 & $32.3^{*}$ & .074 & .271 & -.27 & .79 \\
\hline Temporal $^{\mathrm{a}}$ & .44 & 8 & 126 & .087 & .27 & .61 & $5.1^{*}$ & 19.3 & $31.4^{*}$ & .044 & .211 & .03 & .85 \\
\hline Unilateral left & .56 & 20 & 305 & .044 & .48 & .65 & $12.9^{*}$ & 31.6 & $88.2^{*}$ & .027 & .166 & .24 & .89 \\
\hline Unilateral right & .37 & 23 & 382 & .036 & .30 & .44 & $10.1^{*}$ & 13.4 & $44.3^{*}$ & .014 & .118 & .14 & .60 \\
\hline \multicolumn{14}{|c|}{ Semantic fluency } \\
\hline Frontal, total & .54 & 23 & 255 & .032 & .48 & .61 & $16.8^{*}$ & 29.2 & 32.9 & .008 & .087 & .37 & .71 \\
\hline Unilateral left & .66 & 6 & 48 & .048 & .56 & .75 & $13.6^{*}$ & 43.2 & 5.5 & .001 & .035 & .59 & .73 \\
\hline Unilateral right & .48 & 6 & 47 & .062 & .35 & .60 & $7.7 *$ & 22.6 & 2.7 & .000 & .000 & .48 & .48 \\
\hline Nonfrontal, total & .55 & 7 & 74 & .079 & .39 & .70 & $7.0 *$ & 30.2 & $16.5^{*}$ & .025 & .158 & .24 & .86 \\
\hline Unilateral left & .64 & 4 & 36 & .087 & .47 & .82 & $7.4^{*}$ & 41.4 & 6.1 & .015 & .122 & .40 & .88 \\
\hline Unilateral right & .44 & 3 & 38 & .118 & .21 & .67 & $3.7 *$ & 19.2 & 4.0 & .021 & .145 & .15 & .72 \\
\hline Temporal & .61 & 5 & 60 & .083 & .44 & .77 & $7.3 *$ & 36.7 & $10.3^{*}$ & .019 & .139 & .33 & .88 \\
\hline Unilateral left & .67 & 11 & 92 & .035 & .60 & .74 & $18.9 *$ & 44.9 & 11.6 & .001 & .043 & .59 & .75 \\
\hline Unilateral right & .47 & 13 & 176 & .038 & .39 & .54 & $12.3^{*}$ & 21.7 & 8.6 & .000 & .000 & .47 & .47 \\
\hline
\end{tabular}

Note. $\quad \mathrm{CI}=$ confidence interval. $P V=$ percentage of variance.

${ }^{\text {a }}$ In this group, $97 \%$ of the patients had focal temporal lesions (i.e., 122 of 126 patients).

$* p<.05$.

indexed by the $P V$ accounted for by group membership, nonfrontal and temporal patients are substantially more impaired on semantic than on phonemic fluency.

For phonemic fluency, significant heterogeneity was associated with all the mean effects with the exception of that for the unilat-

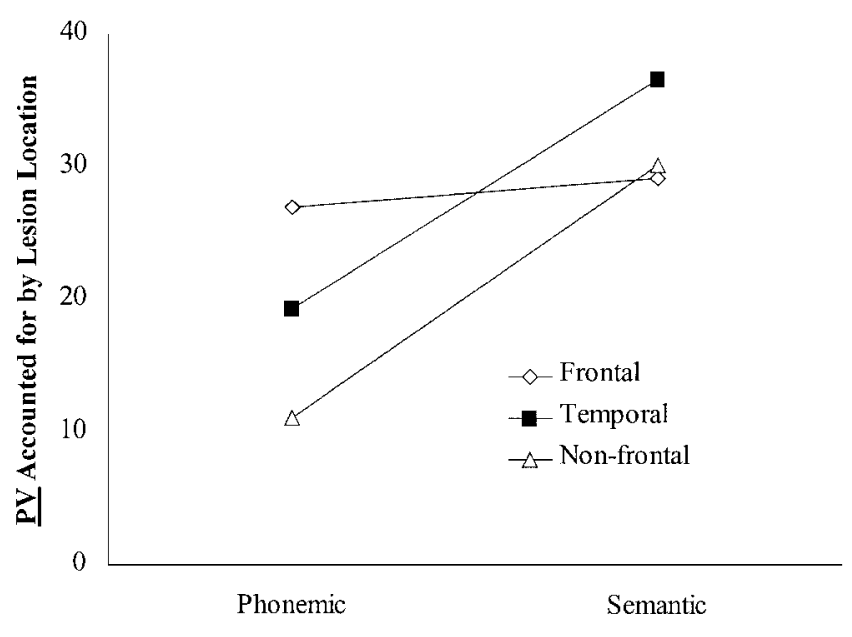

Fluency Type

Figure 1. Percentage of variance $(P V)$ accounted for on phonemic versus semantic fluency performance by the presence of focal frontal, nonfrontal, or specifically, temporal cortical lesions. eral right frontal group. However, compared with frontal patients, the mean effects for nonfrontal and, specifically, temporal patients were associated with substantially greater heterogeneity; for the frontal groups, $\sigma_{\theta}^{2}$ ranged from .009 to .013 ; for nonfrontal and specifically temporal patients, the corresponding values were from .044 to .074 .

For semantic fluency, the studies contributing to the mean effect for the total nonfrontal and temporal groups are significantly heterogeneous as indexed by $Q$, although again, not to the same degree as the total nonfrontal, unilateral left nonfrontal, unilateral right nonfrontal, and temporal groups on phonemic fluency. For semantic fluency, the effects contributing to each mean for all other groups are statistically homogeneous.

\section{Testing for Publication Bias}

A number of validity threats have been identified that may lead to imprecise conclusions in both nonquantitative and meta-analytic reviews. Particularly problematic is "the file drawer problem," which refers to the fact that significant results are more likely to be published than nonsignificant results (Easterbrook, Berlin, Gopalan, \& Mathews, 1991). To assess whether this bias posed a threat to the results of the current study, funnel plot diagrams were constructed for both phonemic and semantic fluency. These diagrams plot sample size against the corresponding study-level effect; if nonsignificant results have been discriminated against, there should be a relative absence of studies with small sample sizes that report weak effects. There was no evidence of this bias operating for either type of fluency. 


\section{Phonemic Fluency Relative to Other Cognitive Measures}

Table 2 presents study-level effects for VIQ-FSIQ, TMT-A, WCST-CC, and WCST-PE alongside the corresponding effects for phonemic fluency for frontal and nonfrontal patients. The mean effect is also presented for each nonfluency variable.

As noted earlier, because there were relatively few effects for each of the nonfluency measures of interest, when comparing phonemic fluency with each, the mean effect for phonemic fluency was recalculated using only those studies that assessed both measures. Thus, 10 studies assessed performance on both IQ and phonemic fluency in frontal patients; in addition to calculating a mean effect for IQ from these 10 studies (which was .18, as shown in Table 2), the mean effect for phonemic fluency was recalculated using only these same 10 studies. The mean phonemic fluency effects used in comparisons against IQ, TMT-A, WCST-CC, and WCST-PE were $.48(K=10), .31(K=4), .58(K=3)$, and .58 $(K=3)$, respectively, for frontal patients and $.21(K=3), .13$ $(K=1), .26(K=1)$, and $.26(K=1)$, respectively, for nonfrontal patients.

The $P V$ accounted for by group membership for frontal and nonfrontal patients relative to healthy controls is presented in Figure 2 for each of the nonfluency measures as well as for phonemic fluency. For studies that assessed both IQ and phonemic fluency in frontal patients $(K=10)$, in terms of the $P V$ accounted for by group membership (i.e., frontal patients vs. healthy controls), the phonemic fluency deficit $(P V=22.7 \%)$ qualified as a differential deficit relative to IQ $(P V=3.3 \% ; \Delta P V=19.4 \%)$. However, for studies that assessed both IQ and phonemic fluency in nonfrontal patients $(K=3)$, this was not the case; $P V$ accounted for by group membership $=4.6 \%$ and $3.8 \%$, respectively;
$\Delta P V=0.8 \%$. Although only one study compared patients with specifically temporal lesions on phonemic fluency and FSIQ, the difference in the $P V$ accounted for by group membership on these two measures was also small (2.4\%; Stanhope, Guinan, \& Kopelman, 1998).

For frontal patients $(K=4)$, again, in terms of the $P V$ accounted for by group membership the phonemic fluency deficit $(P V=9.9 \%)$ qualified as a differential deficit relative to TMT-A $(P V=3.4 \%$; $\Delta P V=6.5 \%)$. For patients with nonfrontal damage $(K=1)$, although it is unfortunate that only one study assessed both phonemic fluency and TMT-A performance in relation to this type of lesion, in this study there was virtually no difference in the $P V$ accounted for by group membership in terms of performance on phonemic fluency relative to TMA $(P V=1.7 \%$ vs. $1.0 \% ; \Delta P V=0.7 \%)$.

For studies that assessed WCST-CC, WCST-PE, and phonemic fluency in frontal patients $(K=3)$, the $P V$ accounted for by group membership for each of these measures was $16.2 \%, 24.7 \%$, and $34.0 \%$, respectively. Thus, although all three measures were very sensitive to the presence of focal frontal lesions, phonemic fluency was notably the most sensitive. However, to address the specificity of each of these measures to focal frontal injuries, it was also necessary to assess their sensitivity to nonfrontal damage. For nonfrontal patients, the $P V$ accounted for by group membership for WCST-CC, WCST-PE, and phonemic fluency was $3.2 \%, 6.8 \%$, and $6.8 \%$, respectively. Thus, for frontal relative to nonfrontal patients, the difference in the $P V$ accounted for by group membership for WCST-CC, WCST-PE, and phonemic fluency was $13.0 \%, 17.9 \%$, and $27.2 \%$, respectively. Therefore, in addition to being more sensitive to frontal injury than either the WCST-CC or WCST-PE, phonemic fluency is also associated with a greater degree of specificity.

Table 2

Effect Sizes for Phonemic Fluency Relative to VIQ, FSIQ, TMT-A, WCST-CC, and WCST-PE, Stratified According to Lesion Location

\begin{tabular}{|c|c|c|c|c|c|c|c|c|}
\hline Study & Laterality & Location & $N$ & VIQ-FSIQ & Phonemic & TMT-A & WCST-CC & WCST-PE \\
\hline \multicolumn{9}{|c|}{ Frontal } \\
\hline Miller (1984) & Left & Frontal & 15 & .14 & .52 & & & \\
\hline Tucha et al. (1999) & Left & Frontal & 45 & .24 & .47 & .06 & & \\
\hline Tucha et al. (1999) & Right & Frontal & 50 & .13 & .23 & .02 & & \\
\hline Miller (1984) & Right & Frontal & 15 & .15 & .42 & & & \\
\hline Butler et al. (1993) & Mixed & Frontal & 17 & $.08^{\mathrm{a}}$ & .38 & & & \\
\hline Owen et al. (1990) & Mixed & Frontal & 19 & .28 & .55 & & & \\
\hline Stanhope et al. (1998) & Mixed & Frontal & 15 & .00 & .52 & & .44 & .53 \\
\hline Stefanova et al. (2002) & Mixed & Frontal & 30 & .11 & .62 & & .41 & .47 \\
\hline Gershberg \& Shimamura (1995) & Mixed & DLPFC & 7 & $.48^{\mathrm{a}}$ & .53 & & .27 & .51 \\
\hline Jurado et al. (2000) & Mixed & Frontal & 13 & .27 & .38 & & & \\
\hline Channon \& Crawford (2000) & Left & Frontal & 6 & & .43 & .39 & & \\
\hline Channon \& Crawford (2000) & Right & Frontal & 13 & & .09 & .24 & & \\
\hline Random-effects weighted mean $r$ & Mixed & Frontal & & .18 & & .18 & .40 & .50 \\
\hline \multicolumn{9}{|c|}{ Nonfrontal } \\
\hline Miller (1984) & Left & Posterior & 15 & .19 & .22 & & & \\
\hline Miller (1984) & Right & Posterior & 15 & .19 & .17 & & & \\
\hline Stanhope et al. (1998) & Mixed & Temporal & 14 & .21 & .26 & & .18 & .26 \\
\hline Channon \& Crawford (2000) & Mixed & Nonfrontal & 12 & & .13 & .10 & & \\
\hline Random-effects weighted mean $r$ & Mixed & Nonfrontal & & .20 & & .10 & .18 & .26 \\
\hline
\end{tabular}

Note. $\quad$ VIQ = verbal IQ; FSIQ = full-scale IQ; TMT-A = Trail Making Test-Part A; WCST = Wisconsin Card Sorting Test; CC = categories completed; $\mathrm{PE}=$ perseverative errors; $\mathrm{DLPFC}=$ dorsolateral prefrontal cortex.

${ }^{\mathrm{a}}$ Effect size calculated from WAIS-R vocabulary. 


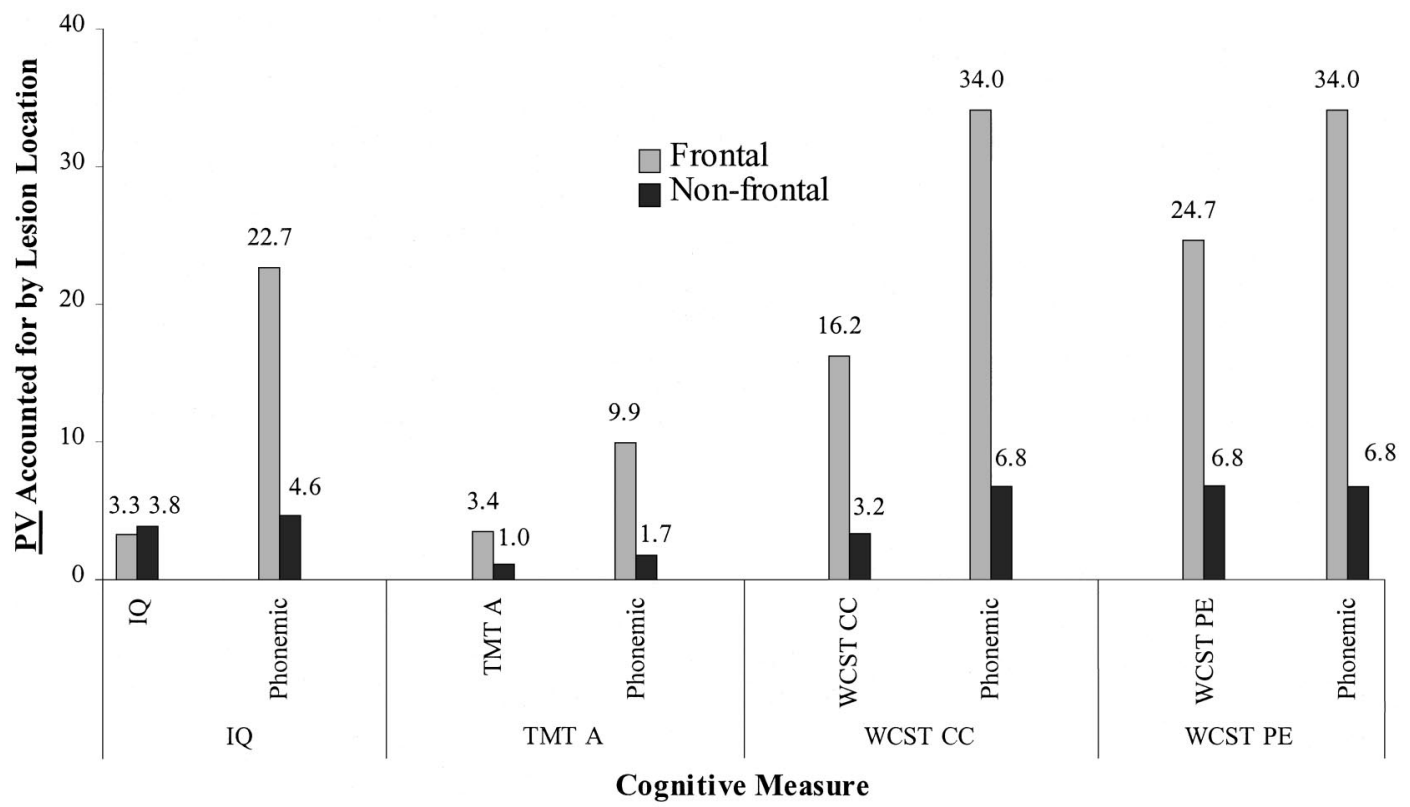

Figure 2. Percentage of variance $(P V)$ accounted for by group membership for phonemic fluency relative to IQ, Trail Making Test-Part A (TMT-A), Wisconsin Card Sorting Test—categories completed (WCST-CC), and Wisconsin Card Sorting Test-perseverative errors (WCST-PE).

\section{Discussion}

\section{Quantifying Verbal Fluency Deficits as a Function of Lesion Location and Laterality}

One of the aims of the current study was to quantify, for the first time using meta-analytic techniques, the degree to which focal lesions in different cortical regions are related to performance on tests of phonemic and semantic fluency. The results are particularly useful because they emphasize the magnitude of the effects. Although researchers are urged to report effect sizes for their individual studies (American Psychological Association, 2001), this is still rarely done in practice. This is unfortunate because effect sizes are far more informative than simply reporting whether a particular effect is significant or not. Moreover, because it is possible, using meta-analysis, to integrate effects across studies, the effects reported here can be considered to be very reliable, robust estimates of the corresponding parameters of interest.

Significant mean effects were associated with both fluency measures across all clinical groups, ranging from .26 to .60 $(P V=6.8-35.9 \%)$ for phonemic fluency and .44 to .67 $(P V=19.2-44.9 \%)$ for semantic fluency. Thus, irrespective of lesion location or laterality, cortical insult produces a substantial deficit on both types of verbal fluency. However, there were systematic differences in the magnitude of the effect as a function of lesion location and lateralization. For phonemic fluency, the largest deficit was associated with unilateral left frontal damage $(r=.60)$, followed closely by the unilateral left group $(r=.56)$, which, it should be noted, also included patients with frontal lesions. These results, therefore, provide strong evidence that phonemic fluency is more sensitive to frontal than nonfrontal and left as opposed to right cortical lesions.

Indeed, the phonemic fluency deficit for the total frontal group $(r=.52)$ was significantly in excess of the corresponding deficit for the total nonfrontal group $(r=.33)$, as were the deficits for the left frontal, left nonfrontal, and left focal groups relative to the right frontal, right nonfrontal, and right focal groups, respectively. Thus, these results are consistent with Ramier and Hacean's (1970) suggestion that phonemic fluency performance is mediated by a verbal factor located in the left hemisphere and an executive component that reflects the integrity of the frontal lobes. Although the temporal group $(r=.44)$ was less impaired on phonemic fluency than the total frontal group $(r=.52)$, this difference did not attain significance. However, as is discussed later, frontal, but not nonfrontal or, specifically, temporal, patients were found to exhibit greater impairment on measures of phonemic fluency than on measures that do not impose substantial executive demands (i.e., IQ and TMT-A).

\section{Semantic Fluency Relative to Phonemic Fluency}

The degree to which measures of phonemic and semantic fluency impose comparable demands on executive processes has been questioned (Perret, 1974). However, the current results indicate that the two types of fluency are equivalent in sensitivity to frontal dysfunction, consistent with other evidence that semantic fluency, like phonemic fluency, does tap executive processes. Brain imaging studies during semantic fluency performance, for instance, have shown activation in the dorsolateral prefrontal cortex (Cardebat et al., 1996; Pujol et al., 1996). Moreover, semantic fluency presumably implicates the same executive processes thought to mediate phonemic fluency performance, such as initiation, efficient organization of verbal retrieval and recall, and self-monitoring; the only notable exception is the need to suppress habitual responses in verbal categorical behavior (Perret, 1974). Thus, it may be that this latter aspect of phonemic fluency performance is a less important component of the executive requirements of the task than has previously been presumed. 
However, in addition to being sensitive to frontal damage, semantic fluency is also very sensitive to temporal pathology. Temporal patients were substantially (although not significantly) more impaired on semantic fluency $(r=.61)$ than were frontal patients $(r=.54 ; \Delta P V$ accounted for $=8.1 \%)$. Moreover, temporal patients were significantly more impaired on semantic fluency $(r=.61)$ than on phonemic fluency $(r=.44)$, suggesting that a compromised semantic system is a more important determinant of impaired performance than executive dysfunction. The current results, therefore, provide strong evidence that, relative to phonemic fluency, tests based on semantic criteria are equally sensitive to frontal insult and, contrary to Perret's (1974) contention that tests of phonemic fluency impose greater executive demands, are in fact comparable in this respect. However, semantic fluency is most affected by damage to the semantic system because larger deficits are associated with injury to temporal structures.

\section{Substantive Versus Artifactual Differences?}

A number of the mean effects identified in the current study were associated with nonsignificant estimates of $Q$. Because $Q$ quantifies the degree of heterogeneity between the studies contributing to a particular mean, this is consistent with Hunter and Schmidt's (1990) contention that much of the variability between studies is artifactual. That is, once sampling error was removed, no significant variance between the studies contributing to each of these mean effects remained. However, when $K$ is relatively small, the power to detect heterogeneity is low (Hedges \& Olkin, 1985). Because for many of the mean effects associated with nonsignificant estimates of $Q$ a relatively small number of groups contributed, it may be premature to conclude that the studies contributing to each of these respective means measures a common underlying parameter (i.e., as is well known, one cannot prove the null hypothesis). Thus, all that can be said is that, for some of these effects, the results are consistent with the null hypothesis of homogeneity of effects.

Further, approximately half of the mean effects were associated with significant heterogeneity. Thus, in addition to artifactual variance, there is evidence of substantive differences between studies beyond lesion location and laterality. Differences between studies in terms of cause, chronicity, and lesion laterality may account for these differences, and Stuss et al. (1998) have identified these and other variables as potential moderators of effects. However, much of this variance will be bundled up within rather than between studies, and thus the specific influence of each cannot be explored in the current study (e.g., there were insufficient studies that were restricted only to chronic vs. acute patients). The heterogeneity statistic $Q$ quantifies the degree of heterogeneity between studies but cannot address the degree of heterogeneity within each of the studies contributing to a mean. However, it is recommended that if future primary research breaks down their samples more fully, meta-analysis should be conducted to address those variables that moderate performance on tests of verbal fluency.

\section{Verbal Fluency Relative to Other Cognitive Measures}

As discussed earlier, if phonemic and semantic fluency deficits are to be legitimately regarded as reflecting executive dysfunction, it is important to demonstrate that patients with frontal damage are more impaired on such measures than on measures that impose only minimal executive demands. This was particularly important for VIQ, and indeed, reflecting the verbal nature of these measures, consistently larger deficits were found for patients with left as opposed to right focal lesions.

The weighted difference between phonemic fluency and IQ in terms of the $P V$ accounted for by group membership was quantified for frontal, nonfrontal, and, specifically, temporal patients. For frontal patients, the mean difference was substantially larger than the corresponding difference for both nonfrontal and specifically temporal patients $(\triangle P V=19.4 \%$ relative to $0.8 \%$ and $2.4 \%$, respectively). Therefore, only for frontal patients can phonemic fluency deficits be regarded as differential deficits.

Moreover, for frontal patients, group membership (i.e., frontals vs. controls) accounted for approximately three times more variance in phonemic fluency performance relative to the TMT-A. Thus, psychomotor slowing alone cannot account for the substantial phonemic fluency deficit associated with frontal dysfunction. However, for patients with nonfrontal damage, the phonemic fluency deficit was comparable to the deficit for TMT-A, and this was reflected in the $P V$ accounted for by group membership on the two measures (i.e., they differed by less than $1 \%$ ). Again, this suggests that, although phonemic fluency deficits associated with frontal damage reflect executive dysfunction, where there are nonfrontal injuries, deficits are consistent with more generalized impairment.

Finally, phonemic fluency was more sensitive to frontal damage than both the WCST-CC and WCST-PE ( $r \mathrm{~s}=.58, .40$, and .50 , respectively). It has been noted that WCST-CC, although a widely used measure, has failed to consistently differentiate patients with frontal insult from healthy controls (Axelrod, Goldman, Heaton, \& Curtiss, 1996; Crawford \& Henry, in press; Mountain \& Snow, 1993; R. M. Reitan \& Wolfson, 1994). Although the WCST-PE was more comparable to phonemic fluency in sensitivity to frontal dysfunction than the WCST-CC, the WCST-PE was poorer at differentiating between frontal and nonfrontal insult relative to phonemic fluency, as was the WCST-CC. Thus, phonemic fluency was not only more sensitive but had better specificity for frontal damage because it accounted for substantially more variance in frontal relative to nonfrontal groups than either the WCST-CC or WCST-PE. Conclusions are, however, limited by the fact that only one study to date has simultaneously assessed performance on these three measures in nonfrontal patients, indicating an important area for future research.

\section{Conclusion}

The current results provide strong support for the validity of both phonemic and semantic fluency as executive measures and indicate that the former is more sensitive to frontal dysfunction than either the WCST-CC or WCST-PE and has better specificity. For frontal, but not nonfrontal, patients, the phonemic fluency deficit qualified as a differential deficit. Moreover, the results challenge Perret's (1974) much-cited conclusion that, relative to semantic fluency, phonemic fluency is more sensitive to executive dysfunction because the mean effect sizes for the total frontal group were comparable for the two types of flu- 
ency. However, semantic fluency is more sensitive to temporal than frontal lesions; thus, semantic fluency appears to be most dependent on the integrity of semantic memory rather than executive processes. Thus, because both measures place comparable demands on frontal structures, but semantic fluency is relatively more dependent on temporal structures, it is suggested that comparison of the relative magnitude of deficits on phonemic and semantic fluency may be used to draw inferences regarding the prominence of executive dysfunction and semantic memory dysfunction, respectively, and, when in the context of performance on other cognitive measures, whether these deficits qualify as differential deficits.

\section{References}

Albert, M. L., \& Sandson, J. (1986). Perseveration in aphasia. Cortex, 22, 103-115.

American Psychological Association. (2001). Publication manual of the American Psychological Association (5th ed.). Washington, DC: Author.

Axelrod, B. N., Goldman, R. S., Heaton, R. K., \& Curtiss, G. (1996). Discriminability of the Wisconsin Card Sorting Test using the standardisation sample. Journal of Clinical and Experimental Neuropsychology, 18, 338-342.

Baldo, J. V., \& Shimamura, A. P. (1998). Letter and category fluency in patients with frontal lobe lesions. Neuropsychology, 12, 259-267.

Baldo, J. V., Shimamura, A. P., Delis, D. C., Kramer, J., \& Kaplan, E. (2001). Verbal and design fluency in patients with frontal lobe lesions. Journal of the International Neuropsychological Society, 7, 586-596.

Beldarrain, M. G., Grafman, J., Pascual-Leone, A., \& Garcia-Monco, J. C. (1999). Procedural learning is impaired in patients with prefrontal lesions. Neurology, 52, 1853-1860.

Benton, A. L. (1968). Differential behavioural effects in frontal lobe disease. Neuropsychologia, 6, 53-60.

Bolter, J., Long, C., \& Wagner, M. (1983). The utility of the Thurstone Word Fluency Test in identifying cortical damage. International Journal of Clinical Neuropsychology, 5, 77-82.

Borkowski, J. G., Benton, A. L., \& Spreen, O. (1967). Word fluency and brain damage. Neuropsychologia, 5, 135-140.

Brooks, J., Fos, L. A., Greve, K. W., \& Hammond, J. S. (1999). Assessment of executive function in patients with mild traumatic brain injury. The Journal of Trauma: Injury Infection and Critical Care, 46, 159163.

Butler, R. W., Rorsman, I., Hill, J. M., \& Tuma, R. (1993). The effects of frontal brain impairment on fluency: Simple and complex paradigms. Neuropsychology, 7, 519-529.

Cardebat, D., Demonet, J. F., Viallard, G., Faure, S., Puel, M., \& Celsis, P. (1996). Brain functional profiles in formal and semantic fluency tasks: A SPECT study in normals. Brain and Language, 52, 305-313.

Channon, S., \& Crawford, S. (2000). The effects of anterior lesions on performance on a story comprehension test: Left anterior impairment on a theory of mind-type task. Neuropsychologia, 38, 1007-1017.

Cohen, J. (1977). Statistical power analysis for the behavioral sciences (Rev. ed.). New York: Academic Press.

Cohen, R. A., Kaplan, R. F., Zuffante, P., Moser, D. J., Jenkins, M. A., Salloway, S., \& Wilkinson, H. (1999). Alteration of intention and self-initiated action associated with bilateral anterior cingulotomy. Journal of Neuropsychiatry \& Clinical Neurosciences, 11, 444-453.

Crawford, J. R., Bryan, J., Luszcz, M. A., Obonsawin, M. C., \& Stewart, L. (2000). The executive decline hypothesis of cognitive aging: Do executive deficits qualify as differential deficits and do they mediate age-related memory decline? Aging, Neuropsychology and Cognition, 7, $9-31$.

Crawford, J. R., \& Henry, J. D. (in press). Assessment of executive deficits.
In P. W. Halligan \& N. Wade (Eds.), The effectiveness of rehabilitation for cognitive deficits. London: Oxford University Press.

Crawford, J. R., Moore, J. W., \& Cameron, I. M. (1992). Verbal fluency: A NART-based equation for the estimation of premorbid performance. British Journal of Clinical Psychology, 31, 327-329.

Crawford, J. R., Obonsawin, M. C., \& Bremner, M. (1993). Frontal lobe impairment in schizophrenia: Relationship to intellectual functioning. Psychological Medicine, 23, 787-790.

Crowe, S. F. (1996). The performance of schizophrenic and depressed subjects on tests of fluency: Support for a compromise in dorsolateral prefrontal functioning. Australian Psychologist, 31, 204-209.

De Vreese, L. P., Neri, M., Rubichi, S., \& Salvioli, G. (1996). Grammatical ambiguity resolution in right hemisphere-damaged patients: Evidence from an insertion task. Aphasiology, 10, 801-814.

Easterbrook, P. J., Berlin, J. A., Gopalan, R., \& Mathews, D. R. (1991). Publication bias in clinical research. Lancet, 337, 867-872.

Field, A. P. (2001). Meta-analysis of correlation coefficients: A Monte Carlo comparison of fixed- and random-effects models. Psychological Methods, 6, 161-180.

Fisher, R. A. (1928). Statistical methods for research workers (2nd ed.). London: Oliver \& Boyd.

Flowers, K. A., Robertson, C., \& Sheridan, M. R. (1995). Some characteristics of word fluency in Parkinson's disease. Journal of Neurolinguistics, 9, 33-46.

Gershberg, F. B., \& Shimamura, A. P. (1995). Impaired use of organizational strategies in free-recall following frontal lobe damage. Neuropsychologia, 33, 1305-1333.

Goulet, P., Joanette, Y., Sabourin, L., \& Giroux, F. (1997). Word fluency after a right-hemisphere lesion. Neuropsychologia, 35, 1565-1570.

Gourovitch, M. L., Kirkby, B. S., Goldberg, T. E., Weinberger, D. R., Gold, J. M., Esposito, G., et al. (2000). A comparison of rCBF patterns during letter and semantic fluency. Neuropsychology, 14, 353-360.

Heaton, R. K. (1981). Wisconsin Card Sorting Test (WCST). Odessa, FL: Psychological Assessment Resources.

Hedges, L., \& Olkin, I. (1985). Statistical methods for meta-analysis. New York: Academic Press.

Helmstaedter, C., Gleissner, U., Zentner, J., \& Elger, C. E. (1998). Neuropsychological consequences of epilepsy surgery in frontal lobe epilepsy. Neuropsychologia, 36, 681-689.

Hodges, J. R., Salmon, D. P., \& Butters, N. (1992). Semantic memory impairment in Alzheimer's disease-Failure of access or degraded knowledge. Neuropsychologia, 30, 301-314.

Hunter, J. E., \& Schmidt, F. L. (Eds.). (1990). Methods of meta-analysis: Correcting error and bias in research findings. Newbury Park: CA: Sage.

Hunter, J. E., \& Schmidt, F. L. (1994). Correcting for sources of artificial variation across studies. In H. Cooper \& L. V. Hedges (Eds.), The handbook of research synthesis (pp. 323-336). New York: Russell Sage Foundation.

Hunter, J. E., Schmidt, F. L., \& Jackson, G. B. (1982). Meta-analysis: Cumulating research findings across studies. Beverly Hills, CA: Sage.

Joanette, Y., \& Goulet, P. (1986). Criterion-specific reduction of verbal fluency in right-brain-damaged right-handers. Neuropsychologia, 24, 875-879.

Jurado, M. A., Mataro, M., Verger, K., Bartumeus, F., \& Junque, C. (2000). Phonemic and semantic fluencies in traumatic brain injury patients with focal frontal lesions. Brain Injury, 14, 789-795.

Laws, K. R. (1999). A meta-analytic review of Wisconsin card sort studies in schizophrenia: General intellectual deficit in disguise? Cognitive Neuropsychiatry, 4, 1-35.

Leclercq, M., Couillet, J., Azouvi, P., Marlier, N., Martin, Y., Strypstein, E., \& Rousseaux, M. (2000). Dual task performance after severe diffuse traumatic brain injury or vascular prefrontal damage. Journal of Clinical and Experimental Neuropsychology, 22, 339-350. 
Levine, B., Stuss, D. T., Milberg, W. P., Alexander, M. P., Schwartz, M., $\&$ MacDonald, R. (1998). The effects of focal and diffuse brain damage on strategy application: Evidence from focal lesions, traumatic brain injury and normal aging. Journal of the International Neuropsychological Society, 4, 247-264.

Loring, D. W., Meador, K. J., \& Lee, G. P. (1994). Effects of temporal lobectomy on generative fluency and other language functions. Archives of Clinical Neuropsychology, 9, 229-238.

Luckhurst, L., \& Lloyd-Jones, T. J. (2001). A selective deficit for living things after temporal lobectomy for relief of epileptic seizures. Brain and Language, 79, 266-296.

Mangels, J. A., Ivry, R. B., \& Shimizu, N. (1998). Dissociable contributions of the prefrontal and neocerebellar cortex to time perception. Cognitive Brain Research, 7, 15-39.

Martin, A., Wiggs, C. L., Lalonde, F., \& Mack, C. (1994). Word retrieval to letter and semantic cues: A double dissociation in normal subjects using interference tasks. Neuropsychologia, 32, 1487-1494.

Martin, R. C., Loring, D. W., Meador, K. J., \& Lee, G. P. (1990). The effects of lateralized temporal lobe dysfunction on formal and semantic word fluency. Neuropsychologia, 28, 823-829.

Miller, E. (1984). Verbal fluency as a function of a measure of verbal intelligence and in relation to different types of cerebral pathology. British Journal of Clinical Psychology, 23, 53-57.

Milner, B. (1964). Some effects of frontal lobectomy in man. In J. M. Warren \& K. Akert (Eds.), The frontal granular cortex and behavior (pp. 313-331). New York: McGraw-Hill.

Monsch, A. U., Seifritz, E., Taylor, K. I., Ermini-Funfschilling, D., Stahelin, H. B., \& Spiegel, R. (1997). Category fluency is also predominantly affected in Swiss Alzheimer's disease patients. Acta Neurologica Scandinavica, 95, 81-84.

Mountain, M. A., \& Snow, W. G. (1993). Wisconsin Card Sorting Test as a measure of frontal pathology: A review. The Clinical Neuropsychologist, 7, 108-118.

National Research Council. (1992). Combining information; statistical issues and opportunities for research. Washington, DC: National Academies Press.

Owen, A., Downes, J., Sahakian, B., Polkey, C., \& Robbins, T. (1990). Planning and spatial working memory following frontal lobe lesions in man. Neuropsychologia, 28, 1021-1034.

Parker, D. M., \& Crawford, J. R. (1992). Assessment of frontal lobe function. In J. R. Crawford, D. M. Parker, \& W. W. McKinlay (Eds.), A handbook of neuropsychological assessment (pp. 267-291). London: Erlbaum.

Pendleton, M. G., Heaton, R. K., Lehman, R. A. W., \& Hulihan, D. (1982). Diagnostic utility of the Thurstone Word Fluency Test in neuropsychological evaluations. Journal of Clinical Neuropsychology, 4, 307-317.

Perret, E. (1974). The left frontal lobe of man and the suppression of habitual responses in verbal categorical behaviour. Neuropsychologia, 12, 323-330.

Phillips, L. H. (1997). Do "frontal tests" measure executive function? Issues of assessment and evidence from fluency tests. In P. M. A. Rabbitt (Ed.), Methodology of frontal and executive function (pp. 191-213). Hove, England: Psychology Press.

Pujol, J., Vendrell, P., Deus, J., Kulisevsky, J., Marti-Vilalta, J. L., Garcia, C., et al. (1996). Frontal lobe activation during word generation studied by functional MRI. Acta Neurologica Scandinavica, 93, 403-410.

Ramier, A. M., \& Hecaen, H. (1970). Role respectif des atteintes frontales et de la lateralisation lesionnelle dans les deficits de la "fluence verbale" [The respective role of frontal injuries and lateral lesions on deficits of "verbal fluency"]. Revus Neurologique, 123, 17-22.

Raudenbush, S. W. (1994). Random effects models. In H. Cooper \& L. V. Hedges (Eds.), The handbook of research synthesis (pp. 301-321). New York: Russell Sage Foundation.
Reitan, R. M., \& Wolfson, D. (1994). A selective and critical review of neuropsychological deficits and the frontal lobes. Neuropsychology Review, 4, 161-195.

Reitan, W. (1990). The Halstead-Reitan Neuropsychological Test Battery: Theory and clinical interpretation. Tucson, AZ: Reitan Neuropsychology Labs, University of Arizona.

Rogers, R. D., Sahakian, B. J., Hodges, J. R., Polkey, C. E., Kennard, C., \& Robbins, T. W. (1998). Dissociating executive mechanisms of task control following frontal lobe damage and Parkinson's disease. Brain, $121,815-842$.

Ruff, R. M., Light, R. H., Parker, S. B., \& Levin, H. S. (1997). The psychological construct of word fluency. Brain and Language, 57, 394-405.

Schwartz, S., \& Baldo, J. (2001). Distinct patterns of word retrieval in right and left frontal lobe patients: A multidimensional perspective. Neuropsychologia, 39, 1209-1217.

Shadish, W. R., \& Haddock, C. K. (1994). Combining estimates of effect size. In H. Cooper \& L. V. Hedges (Eds.), The handbook of research synthesis (pp. 261-281). New York: Russell Sage Foundation.

Snedecor, G. W., \& Cochran, W. G. (1989). Statistical methods (8th ed.). Ames: Iowa State University Press.

Stanhope, N., Guinan, E., \& Kopelman, M. D. (1998). Frequency judgements of abstract designs by patients with diencephalic, temporal lobe or frontal lobe lesions. Neuropsychologia, 36, 1387-1396.

Stefanova, E. D., Kostic, V. S., Ziropadja, L., Markovic, M., \& Ocic, G. (2002). Serial position learning effects in patients with aneurysms of the anterior communicating artery. Journal of Clinical and Experimental Neuropsychology, 24, 687-694.

Stuss, D. T., Alexander, M. P., Hamer, L., Palumbo, C., Dempster, R., Binns, M., et al. (1998). The effects of focal anterior and posterior brain lesions on verbal fluency. Journal of the International Neuropsychological Society, 4, 265-278.

Stuss, D. T., Alexander, M. P., Palumbo, C. L., Buckle, L., Sayer, L., \& Pogue, J. (1994). Organizational strategies of patients with unilateral or bilateral frontal lobe injury in word list learning tasks. Neuropsychology, 8, 355-373.

Stuss, D. T., \& Benson, D. F. (1986). The frontal lobes. New York: Raven Press.

Stuss, D. T., Craik, F. I. M., Sayer, L., Franchi, D., \& Alexander, M. P. (1996). Comparison of older people and patients with frontal lesions: evidence from word list learning. Psychology and Aging, 11, 387-395.

Stuss, D. T., Gallup, G. G., \& Alexander, M. P. (2001). The frontal lobes are necessary for "theory of mind." Brain, 124, 279-286.

Stuss, D. T., Toth, J. P., Franchi, D., Alexander, M. P., Tipper, S., \& Craik, F. I. M. (1999). Dissociation of attentional processes in patients with focal frontal and posterior lesions. Neuropsychologia, 37, 1005-1027.

Sylvester, C. Y. C., \& Shimamura, A. P. (2002). Evidence for intact semantic representations in patients with frontal lobe lesions. Neuropsychology, 16, 197-207.

Terry, R. D., \& Katzman, R. (1983). Senile dementia of the Alzheimer type. Annals of Neurology, 14, 497-506.

Thomson, A. M., Taylor, R., \& Whittle, I. R. (1998). Assessment of communication impairment and the effects of resective surgery in solitary, right-sided supratentorial intracranial tumours: A prospective study. British Journal of Neurosurgery, 12, 423-429.

Thurstone, L. L., \& Thurstone, T. G. (1941). Factorial studies of intelligence. Psychometric Monographs, 2, 94.

Troyer, A. K., Moscovitch, M., Winocur, G., Alexander, M. P., \& Stuss, D. (1998). Clustering and switching on verbal fluency: the effects of focal frontal- and temporal-lobe lesions. Neuropsychologia, 36, 499-504.

Tucha, O., Smely, C., \& Lange, K. W. (1999). Verbal and figural fluency in patients with mass lesions of the left or right frontal lobes. Journal of Clinical and Experimental Neuropsychology, 21, 229-236. 
Varley, R. (1995). Lexical-semantic deficits following right hemisphere damage: Evidence from verbal fluency tasks. European Journal of Disorders of Communication, 30, 362-371.

Wechsler, D. (1955). WAIS manual. New York: Psychological Corporation.

Wechsler, D. (1981). WAIS-R manual. New York: Psychological Corporation.
Welsh, M. C., Satterlee-Cartmell, T., \& Stine, M. (1999). Towers of Hanoi and London: Contribution of working memory and inhibition to performance. Brain and Cognition, 41, 231-242.

Wertz, R. T., Dronkers, N. F., \& Shubitowski, Y. (1986). Discriminant function analysis of performance by normals and left hemisphere, right hemisphere, and bilaterally brain damaged patients on a word fluency measure. Clinical Aphasiology, 16, 257-266.

\section{Appendix}

Studies Included in Quantitative Review

\begin{tabular}{|c|c|c|c|c|c|c|}
\hline Study & Lesion laterality & Lesion location & $N$ & Type of lesion & $\mathrm{PF} r$ & $\mathrm{SF} r$ \\
\hline Baldo et al. (2001) & Left & Frontal & 6 & Unilateral (mixed causes) & .81 & .79 \\
\hline Troyer et al. (1998) & Left & Dorsolateral & 14 & Unilateral (mixed causes) & .73 & .63 \\
\hline Baldo \& Shimamura (1998) & Left & Frontal & 6 & Unilateral (mixed causes) & .72 & .67 \\
\hline Helmstaedter et al. (1998) & Left & Frontal & 17 & Unilateral (surgery) & .68 & \\
\hline Stuss et al. (1994) & Left & Frontal & 5 & Unilateral (mixed causes) & .64 & \\
\hline Stuss et al. (1996) ${ }^{a}$ & Left & Frontal & 10 & Unilateral (not specified) & .61 & .69 \\
\hline Stuss et al. (1999) & Left & Frontal & 6 & Unilateral (mixed causes) & .55 & .49 \\
\hline Miller (1984) & Left & Frontal & 15 & Unilateral (most neoplastic) & .52 & \\
\hline Rogers et al. (1998) & Left & Frontal & 6 & Unilateral (mixed causes) & .49 & .37 \\
\hline Tucha et al. (1999) & Left & Frontal & 45 & Unilateral (tumor) & .47 & \\
\hline Pendleton et al. (1982) & Left & Frontal & 20 & Unilateral (mixed causes) & .47 & \\
\hline Channon \& Crawford (2000) & Left & Frontal & 6 & Unilateral (mixed causes) & .43 & \\
\hline Helmstaedter et al. (1998) & Right & Frontal & 16 & Unilateral (surgery) & .63 & \\
\hline Baldo et al. (2001) & Right & Frontal & 5 & Unilateral (mixed causes) & .58 & .59 \\
\hline Baldo \& Shimamura (1998) & Right & Frontal & 6 & Unilateral (mixed causes) & .55 & .52 \\
\hline Rogers et al. (1998) & Right & Frontal & 6 & Unilateral (mixed causes) & .43 & .20 \\
\hline Miller (1984) & Right & Frontal & 15 & Unilateral (most neoplastic) & .42 & \\
\hline Stuss et al. (1994) & Right & Frontal & 7 & Unilateral (mixed causes) & .41 & \\
\hline Pendleton et al. (1982) & Right & Frontal & 23 & Unilateral (mixed causes) & .37 & \\
\hline Stuss et al. $(1996)^{\mathrm{a}}$ & Right & Frontal & 9 & Unilateral (not specified) & .37 & .36 \\
\hline Stuss et al. (1999) & Right & Frontal & 10 & Unilateral (mixed causes) & .34 & .51 \\
\hline Troyer et al. (1998) & Right & Dorsolateral & 11 & Unilateral (mixed causes) & .34 & .50 \\
\hline Tucha et al. (1999) & Right & Frontal & 50 & Unilateral (tumor) & .23 & \\
\hline Channon \& Crawford (2000) & Right & Frontal & 13 & Unilateral (mixed causes) & .09 & \\
\hline Stuss et al. (1999) & Bilateral & Frontal & 6 & Bilateral (mixed causes) & .55 & .55 \\
\hline Stuss et al. (1994) & Bilateral & Frontal & 11 & Bilateral (mixed causes) & .55 & \\
\hline Stuss et al. (1996) ${ }^{a}$ & Bilateral & Frontal & 13 & Bilateral (not specified) & .50 & .62 \\
\hline R. A. Cohen et al. (1999) & Bilateral & Anterior & 18 & Bilateral (surgery) & & .32 \\
\hline Troyer et al. (1998) & Mixed & Medial frontal & 17 & Uni-, bilateral (mixed causes) & .66 & .48 \\
\hline Leclercq et al. (2000) & Mixed & Frontal & 9 & Uni-, bilateral (aneurysms) & .63 & .71 \\
\hline Stefanova et al. (2002) & Mixed & Frontal & 30 & Uni-, bilateral (aneurysms) & .62 & .29 \\
\hline Owen et al. (1990) & Mixed & Frontal & 19 & Uni-, bilateral (surgery) & .55 & .42 \\
\hline Gershberg \& Shimamura (1995) & Mixed & Dorsolateral & 7 & Unilateral (cerebral vascular) & .53 & \\
\hline Stanhope et al. (1998) & Mixed & Frontal & 15 & Uni-, bilateral (mixed causes) & .52 & \\
\hline Butler et al. (1993) & Mixed & Frontal & 17 & Uni-, bilateral (tumor) & .38 & \\
\hline Jurado et al. (2000) & Mixed & Frontal & 13 & Uni-, bilateral (TBI) & .38 & .29 \\
\hline Troyer et al. (1998) & Mixed & Medial frontal & 11 & Uni-, bilateral (mixed causes) & .30 & .44 \\
\hline Schwartz \& Baldo (2001) & Mixed & Frontal & 13 & Uni-, bilateral (mixed causes) & & .64 \\
\hline Sylvester \& Shimamura (2002) & Mixed & Dorsolateral & 11 & Unilateral (mixed causes) & & .67 \\
\hline R. C. Martin et al. (1990) & Left & Temporal & 15 & Unilateral (surgery) & .74 & .79 \\
\hline Helmstaedter et al. (1998) & Left & Temporal & 24 & Unilateral (surgery) & .55 & \\
\hline Stuss et al. (1999) & Left & Posterior & 7 & Unilateral (mixed causes) & .53 & .51 \\
\hline Troyer et al. (1998) & Left & Temporal & 9 & Unilateral (mixed causes) & .30 & .63 \\
\hline Pendleton et al. (1982) & Left & Nonfrontal & 22 & Unilateral (mixed causes) & .24 & \\
\hline Miller (1984) & Left & Posterior & 15 & Unilateral (mostly neoplastic) & .22 & \\
\hline Luckhurst \& Lloyd-Jones (2001) & Left & Temporal & 5 & Unilateral (surgery) & & .40 \\
\hline R. C. Martin et al. (1990) & Right & Temporal & 17 & Unilateral (surgery) & .61 & .61 \\
\hline Helmstaedter et al. (1998) & Right & Temporal & 21 & Unilateral (surgery) & 60 & \\
\hline Pendleton et al. (1982) & Right & Nonfrontal & 17 & Unilateral (mixed causes) & .21 & \\
\hline Miller (1984) & Right & Posterior & 15 & Unilateral (mostly neoplastic) & .17 & \\
\hline Troyer et al. (1998) & Right & Temporal & 14 & Unilateral (mixed causes) & .10 & .34 \\
\hline Stuss et al. (1999) & Right & Posterior & 7 & Unilateral (mixed causes) & -.29 & .24 \\
\hline Stanhope et al. (1998) & Mixed & Temporal & 14 & Uni-, bilateral (mixed causes) & .26 & \\
\hline Channon \& Crawford (2000) & Mixed & Most temporal & 12 & Unilateral (mixed causes) & .13 & \\
\hline Wertz et al. (1986) & Left & - & 40 & Unilateral (cerebrovascular) & .80 & \\
\hline De Vreese et al. (1996) & Left & Mixed & 8 & Unilateral (ischemia) & .65 & .64 \\
\hline
\end{tabular}


Appendix (continued)

\begin{tabular}{|c|c|c|c|c|c|c|}
\hline Study & Lesion laterality & Lesion location & $N$ & Type of lesion & $\mathrm{PF} r$ & $\mathrm{SF} r$ \\
\hline Pendleton et al. (1982) & Left & Mixed & 16 & Unilateral (mixed causes) & .41 & \\
\hline Albert \& Sandson (1986) & Right & - & 13 & Unilateral (cause not specified) & .57 & \\
\hline Wertz et al. (1986) & Right & - & 40 & Unilateral (cerebral vascular) & .38 & \\
\hline Goulet et al. (1997) & Right & - & 15 & Unilateral (stroke) & .32 & .35 \\
\hline Pendleton et al. (1982) & Right & Mixed & 16 & Unilateral (mixed causes) & .28 & \\
\hline Joanette \& Goulet (1986) & Right & Mixed & 35 & Unilateral (vascular accident) & .18 & .47 \\
\hline De Vreese et al. (1996) & Right & Mixed & 8 & Unilateral (ischemia) & .17 & .55 \\
\hline Varley (1995) & Right & - & 20 & Unilateral (vascular lesions) & & $.25^{\mathrm{b}}$ \\
\hline Thomson et al. (1998) & Right & Mixed & 33 & Unilateral (tumor) & & .36 \\
\hline
\end{tabular}

Note. $\mathrm{PF}=$ phonemic fluency; $\mathrm{SF}=$ semantic fluency; $\mathrm{TBI}=$ traumatic brain injury.

a This study provides data for three different control groups; the middle-aged group was used to derive effect sizes because it most closely matched the age of the patients. ${ }^{b}$ Mean effect size collapsed across different measures of semantic fluency. 